\title{
Conformal Radiation
}

a report by

Eric C Rost, MD

Director, Nikki Lynn TomoTherapy Program, Southeast Regional Cancer Center of Tallahassee and

Chairman and Founder, CancerHope Foundation

DOI: 10.17925/OHR.2005.00.00.1k

\section{Introduction}

Conformal therapy is the most dramatic advance in the past 10 years of clinical oncology. It involves accurate delivery of effective radiation to produce cancer control. It limits the side effects and complications associated with less sophisticated methods of radiation. The minimum requirements for conformal therapy are a computed tomography (CT) data set to produce a virtual three dimensional (3-D) view of the patient, treatment planning software, and a delivery system capable of producing radiation and verifying accurate delivery. The use of conformal radiation allows for fewer side effects, better results of treatment, and the ability to perform salvage treatment for recurrence in a much safer manner than previously. There are several types of conformal therapy; the more advanced the technique, the better result of treatment.

The goal of radiation treatment has always been to destroy the cancer without harming normal structures and cells. Normal structure tolerance is the greatest limitation of successful treatment. In order to accomplish this goal, treatment is delivered in discreet fractions or applications that allow for the maximum radiobiological effect to destroy cancer cells but spare normal cells in the areas of treatment. This is why radiation is delivered over several days rather than in a single visit. However, the application of a radiation plan must account for verification of each treatment to assure proper targeting over the entire course of therapy. The ability to reliably reproduce the planned treatment is a mandatory function of any radiation delivery system. Radiation is very much a form of molecular surgery with a goal to eliminate only the undesirable tissue. Radiation has a greater ability to effectively rid an area of tumor than traditional surgery because, when done correctly, radiation causes less tissue disturbance than surgery. In addition, it is often anatomically impossible to remove cancer from a vital organ or tissue with surgery alone. Radiation treatment allows for more accurate differentiation between diseased and healthy tissues. The ultimate goal is to have a single beam treat each individual cell. Conformal radiation paves the way to precise treatment delivery.
Non-conformal or Traditional Therapy

Traditional radiation is limited to between two and four ports of entry. The shape of the fields and the depth of penetration can be manipulated, but that is the limit of the ability to conform the radiation. It does not allow for any specific conformation to avoid normal structures in the 'box' - the resulting area of treatment is a homogenous region of radiation. The treatment is therefore limited by the normal structure's radiation tolerance. The treatment area can be moderated over time, but the entire plan is still delivered based on the acceptable dose limits of the normal tissues involved. This is the cause of the side effects associated with radiation. If more beams are added from different directions, a more defined target region can be established. However, the accuracy of the plan is always limited because the correct location of the target region or surrounding normal tissue cannot be verified. Advanced computer planning and visualization are needed to improve accuracy.

\section{Simple Conformal Therapy}

Simple conformal therapy begins with a virtual reconstruction of the area of concern using CT scanning. Normal tissue and other avoidance structures are identified as well as the target. The importance of seeing the relationship of each structure cannot be overemphasized. This 3-D view allows the planning of fields from multiple planes to minimize normal tissue involvement. Radiation beams are then designed to maximize the dose to the target and limit the critical structure exposure. In the simplest form of conformal therapy, five to seven beams are used to focus the radiation on the tissue from multiple angles. The cumulative dose deposited will be effective for killing the cancer cells, but avoids the majority of critical structures by spreading out the dose into multiple regions. Biologically, a smaller amount of radiation to a larger region allows for less normal tissue damage.

The limiting factors in simple conformal therapy are the calculating capability of the planning system and the ability of the treatment equipment to verify and deliver the plan successfully and accurately. However, simple

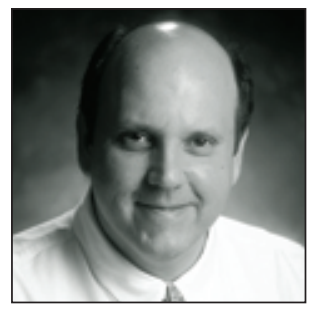

Eric C Rost, MD, is the Director of the Nikki Lynn TomoTherapy Program at the Southeast Regional Cancer Center of Tallahassee Florida. His area of specialty is TomoTherapy radiation. He is also a board-certified oncologist and Chairman and Founder of the CancerHope Foundation in Florida - a not-for-profit foundation for patients and families with cancer. Dr Rost has been practicing clinical oncology for more than 15 years. He graduated from Northeastern Ohio Universities College of Medicine and received his training at Johns Hopkins and ColumbiaPresbyterian Hospitals. 
Figure I:TomoTherapy Daily Image Set for Positional Verification in the Prostate
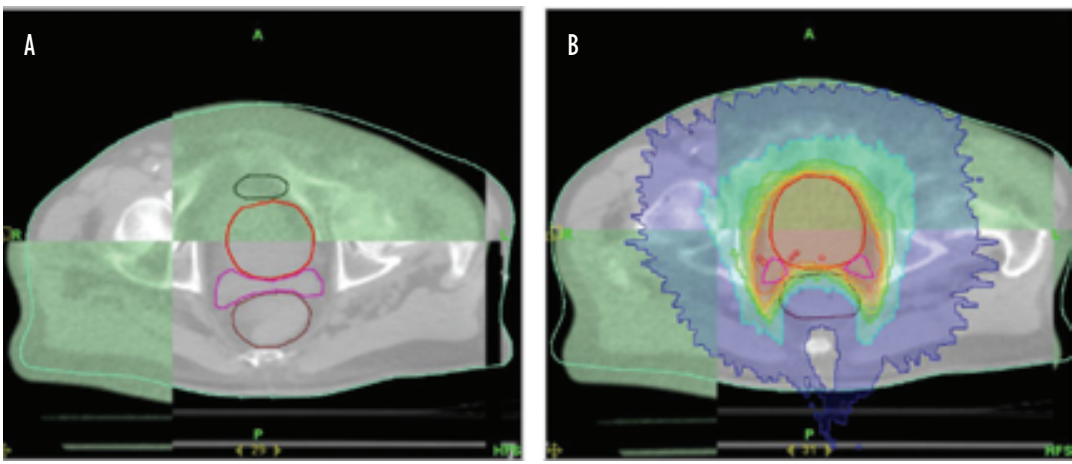

Figure $A$ demonstrates the variance in set up position by overlay of planning data set with the daily Tomolmage (green). Note the misalignment of pelvic bones and prostate. Figure $B$ is the approved and adjusted position with the dose cloud applied. Although this figure is a single view, the complete data set in three dimensions is used in daily verification.

conformal therapy does allow significant advantage over traditional therapy, especially for those tumors in close association with vital structures.

\section{Intensity Modulated Radiation Therapy}

Intensity modulated radiation therapy (IMRT) is the next evolution of conformal radiation delivery. It modifies the treatment by weighting the doses of each of the five to seven fields to produce desired accumulations. It is a dynamic type of therapy that effectively divides each main field into many smaller beams. IMRT uses dynamic leaves or blocks within each field to deliver different amounts of radiation within the main field. It is similar to breaking down the fields into a set of voxels determined by the width and movement capacity of the blocking leaves. The resulting effect is to produce hundreds of segments within the main set of beams. The composite dose plan of IMRT does allow for significant sparing of normal tissue unavailable with simple conformal therapy. This modulated conformal therapy is very specific. However, it is also very complicated to account for so many segmented interactions in treatment planning. This highly conformal method makes the daily variations in position of the target and normal tissue rather important. Margins are included to compensate for physiologic motions, but special immobilization devices are also needed to ensure accuracy. These devices can be very time-consuming to apply. They can also be very unpleasant for the patient and add potential complications and cost to the treatment.

Some of the limitations of IMRT are the amount of time it takes to calculate the plan as well as the time it takes to deliver each treatment. Often, the regions of treatment are too large to safely treat with IMRT, as the integral dose delivered to the entire exposed portion is too large. Very tight tumor settings are also limited as physiologic motion cannot be verified without primary daily visualization. This method of delivery is also extensive in planning and quality assurance. Accurate dose delivery is complicated by the lack of direct visualization of the plan in the patient. Instead, a secondary source of measurement (phantoms) is relied on before patient exposure. The data from this assurance method is secondary. IMRT requires great skill in positioning the patient each day. Concern for movement day to day, as well as during treatment, is high because of potential miss in tumor delivery. There is a very tight margin for error. The expertise of the staff required to carry out standard IMRT is higher than other treatment methods, which limits its application in most community settings.

Tomotherapy

TomoTherapy is the latest form of conformal therapy. This is a linear accelerator that is joined to a CT scanning platform, allowing a full 360 degree range of treatment. The radiation is delivered in a dynamic helical form so that very small changes to the radiation field can be produced in a dynamic fashion. The size of the beam width and the ability to shape the fields are accomplished by the helical distribution of radiation and the binary multileaf collimator. The resulting accumulation of dose can be very finely modified. The best way to visualize the TomoTherapy treatment system is to imagine the attack through the eyes of the beam. This beam's eye view can treat if it sees a clear uninterrupted path to the target. If there is critical structure in the path, it does not fire. This allows for a very accurate and fast delivery of multiple 'beamlets'. The number of beamlets from TomoTherapy is an order of magnitude greater than with standard IMRT. TomoTherapy can treat with segmented beamlets typically numbering at 5,000 to 100,000; compared with approximately 150 segments for step-and-shoot IMRT, and 700 segments for sliding-window IMRT. The resulting distribution of dose is the most conformed treatment arrangement available to date. Combined with the treatment capability is the actual visualization capability of megavoltage computer tomography (MVCT) or 'TomoImage'. This function allows for visualization of soft tissues and other structures in 3-D as part of each and every treatment. The accuracy of the overlay comparison and adjustment can be seen in Figure 1 and Figure 2.

The treatment planning for TomoTherapy is unique and specific to the machine. It is an integrated treatment system. However, as accurate and specific as the delivery capabilities of these innumerable beams are, the fact that each and every treatment can be verified makes TomoTherapy the preferred method of 
treatment for any close structure or retreat patient. In fact, the desired dose can be modified each day depending on the position of the patient as well as the position of the target and its related structures. This avoids the mandatory large margins that are built in for safety in all other forms of radiation. The verification of dose and tissue position is very efficient and allows for precise treatment. It also eliminates much of the need for immobilization devices used in other forms of IMRT, thus reducing complications and cost.

TomoTherapy also allows for treatment of multiple treatment sites simultaneously. Traditionally, each anatomically involved area of disease needs to be treated separately, often increasing the total treatment time. With TomoTherapy IMRT, these sites can be safely treated together, thereby reducing the time of treatment by one-half to one-third depending on the situation.

The main advantage of TomoTherapy is the delivery of radiation to very specific regions with greatly reduced side effects. There is no normal tissue tolerance to limit the treatment plan, as has been experienced for decades. In treatment for lung cancer, for instance, not only can the area of tumor and nodal spread in the mediastinum, but the esophageal dose can be reduced, thus sparing the patient any dysphasia traditionally associated with thoracic radiation. It has also been possible to treat the entire pleura and mediastinum in mesothelioma without destruction of the lung. Bilateral acoustic neuromas have been treated simultaneously with no significant exposure to the brain stem or other neural structures. Prostate patients have had a dramatic reduction in bladder and rectal irritation. The treatment thus far has been very successful and the applications for use are still being explored.

Conclusion

Conformal radiation therapy has developed over the years to yield better treatment in many parameters. The greatest facilitator for conformal therapy has been the advancement in computer-generated visualization. This application of advanced imaging to radiation therapy planning and delivery has made these advances possible. Not only is the main goal of tumor eradication closer than it has ever been before, but treatment without side effects and complications has opened new doors for victims of cancer. This has allowed a better outcome and decreased the cost of patient and symptom management.

Although the cost of the systems capable of providing modern types of conformal therapy is initially high, the overall and long-term costs are lower. With higher treatment specificity, a reduction of complications and the number of treatment courses, and the removal of many immobilization devices, is seen.
Figure 2: A Graph of the Position Shifts Needed Each Day for Proper Set-up in One Patient

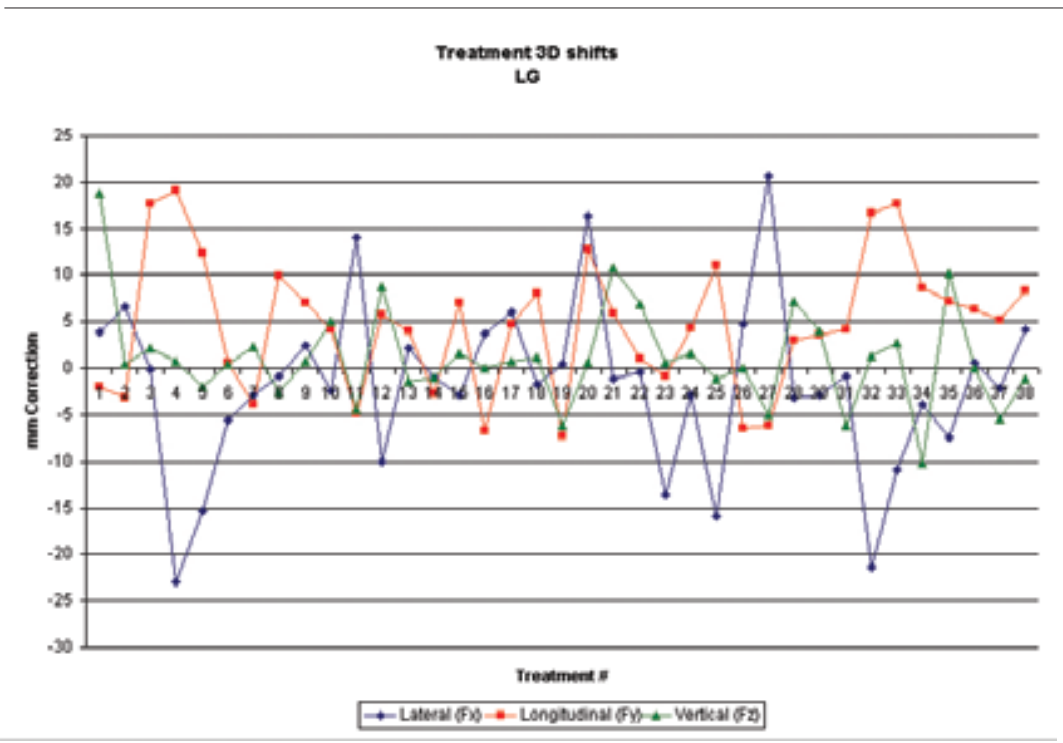

This demonstrates the amount of potential treatment error if daily verification was not part of the integrated process. With image-guided IMRT, the actual position is a straight line, as planned and delivered.

Figure 3: Comparison of the Relative Dose Versus Organ Volume in Pelvic Irradiation for Standard Conformal (Six Field), IMRT, and TomoTherapy IMRT

Bladder Rectum

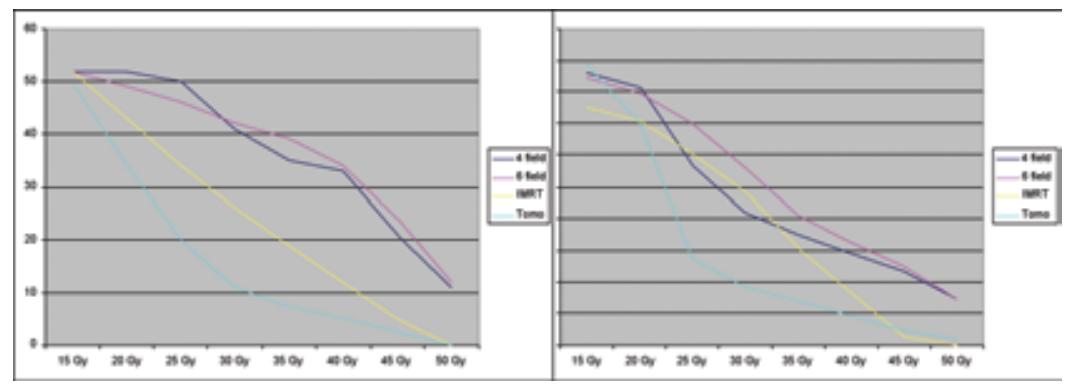

With the introduction of TomoTherapy in the past year, faster tumor responses have been discovered as well as fewer side effects. This is due to increased accuracy in each daily treatment over other forms of conformal therapy. There has also been a decrease in the number of specialized staff needed compared with standard IMRT to safely and accurately deliver treatment. The ability to use an integrated system for IMRT has less synergy problems than standard IMRT. There are seven areas of coordination in all forms of treatment delivery:

- acquisition of the data set;

- structure identification;

- treatment planning;

- verification of treatment plan;

- verification of dose;

- verification of position; and

- treatment delivery. 
TomoTherapy integration combines six of these functions. All other forms of IMRT have separate systems that need to be individually integrated. This advantage eliminates the need for excessive personnel as well as reducing the potential errors associated with each step.

It is no longer necessary to have the cancer patient suffer though caustic and drastic therapies to have a chance for cure or palliation. Tumors that were thought to be untreatable are now easily treated. The side effects with conformal therapy are less than with standard therapies. Older patients and those with serious illnesses can now be treated with every expectation of acceptable results. Coordination with other specialties of oncology, such as surgery and chemotherapy, allows for ever better tumor control and fewer side effects with the use of more advanced forms of radiation delivery than ever before. The age of molecular surgery using radiation has now arrived. The benefit for the patient has never been better. And it is still improving. 